\title{
Concomitant appearance of morphea and vitiligo in a patient with autoimmune thyroiditis
}

\author{
Ahu Yorulmaz', Sevgi Kilic², Ferda Artuz¹, Erhan Kahraman ${ }^{3}$ \\ ${ }^{1}$ Department of Dermatology, Ankara Numune Research and Education Hospital, Ankara, Turkey \\ ${ }^{2}$ Department of Dermatology, Ankara Sincan Dr. Nafiz Korez State Hospital, Ankara, Turkey \\ ${ }^{3}$ Department of Pathology, Ankara Numune Research and Education Hospital, Ankara, Turkey
}

Adv Dermatol Allergol 2016; XXXIII (4): 314-316

DOI: 10.5114/ada.2016.61610

Morphea is a rare fibrosing disorder affecting the skin and underlying tissue. Also called localized scleroderma, morphea is characterized by lesions, which are initially erythematous or dusky violaceous patches eventually turning into hypopigmented sclerotic plaques with a typical lilac-coloured border. Generalized morphea is a rare subtype of morphea characterized by more than four lesions occurring in two or more body sites. Although it has not been fully elucidated yet, it is increasingly perceived that autoimmunity plays the central role in the pathogenesis of both morphea and vitiligo.

Here, we present a case of generalized morphea accompanied by generalized vitiligo in a patient with autoimmune thyroiditis. These three putatively autoimmune diseases in a single patient presumably signify an autoimmune phenomenon in their pathogenesis, which should be further investigated [1-3].

A 62-year-old woman came to our outpatient clinic with a history of two months of purplish discolorations over her trunk. While her family history was unremarkable, past medical history revealed evidence of stable vitiligo, type 2 diabetes mellitus (DM) and autoimmune thyroiditis, which were well-controlled with metformin of $500 \mathrm{mg}$ twice a day and levothyroxine 75 mg once a day. The onset of vitiligo occurred at least 10 years ago, for which she had received topical corticosteroids, topical immunomodulators, systemic corticosteroids, phototherapy and natural home remedies without improvement. Upon dermatological examination we observed multiple depigmented patches over her central face, upper sternal area, upper and lower extremities, especially dorsal aspects of distal parts including hands and foot. We also observed multiple purplish indurated plaques on her torso, scattered over the abdominal area, lower back, left acromial region and right inframammarial area (Figures 1, 2).
Lilac-coloured edge and central hyperpigmentation, which are typical features of plaque type morphea [2] were obvious (Figure 2). Laboratory studies including complete blood count and differential erythrocyte sedimentation rate and urinalysis revealed no abnormalities. A complete serum chemistry profile was within normal limits except high postprandial glucose levels (161 mg/dl (0-140)) and low-density lipoprotein (LDL) cholesterol levels (117 mg/dl (0-100)). Serologic tests for hepatitis B, C, and Borrelia burgdorferi were negative. The thyroid panel was normal, on the other hand anti-thyroglobulin (anti-Tg) and anti-thyroid peroxidase (anti-TPO) values were increased $(21.4 \mathrm{IU} / \mathrm{ml}$ and $814.3 \mathrm{IU} / \mathrm{ml}$, respectively). Anti-nuclear antibody (ANA), anti-double stranded DNA antibody (anti-dsDNA), and antibodies to saline-extracted antigens (ENA panel), and rheumatoid factor were negative. C3, C4 levels, chest X-ray and electrocardiogram were normal. A lesional skin biopsy from the right inframammarial area demonstrated mild focal acanthosis, superficial and deep dermal perivascular lymphocytic infiltrate and focally increased dermal collagen (Figure 3). Based on history, clinical, laboratory and histopathological findings, we made a diagnosis of generalized morphea accompanied by generalized vitiligo. The patient was referred to the endocrinology department for the management of DM and autoimmune thyroiditis. Topical corticosteroid plus topical calcipotriol and systemic antimalarial were prescribed and the patient was followed up.

Morphea, which is a rare chronic disease, encompasses a spectrum of sclerotic dermatosis affecting the skin, subcutaneous tissue and underlying structures, including muscles and bones [1]. Morphea used to be classified into five subtypes: plaque, generalized, bullous, linear and deep according to clinical presentation and disease involvement. On the other hand, another classification

Address for correspondence: Dr. Ahu Yorulmaz, Department of Dermatology, Ankara Numune Research and Education Hospital, Samanpazari, Altindag, 06100 Ankara, Turkey, phone: +90 3125084000, e-mail: ahuyor@gmail.com Received: 7.11.2015, accepted: 27.11.2015. 


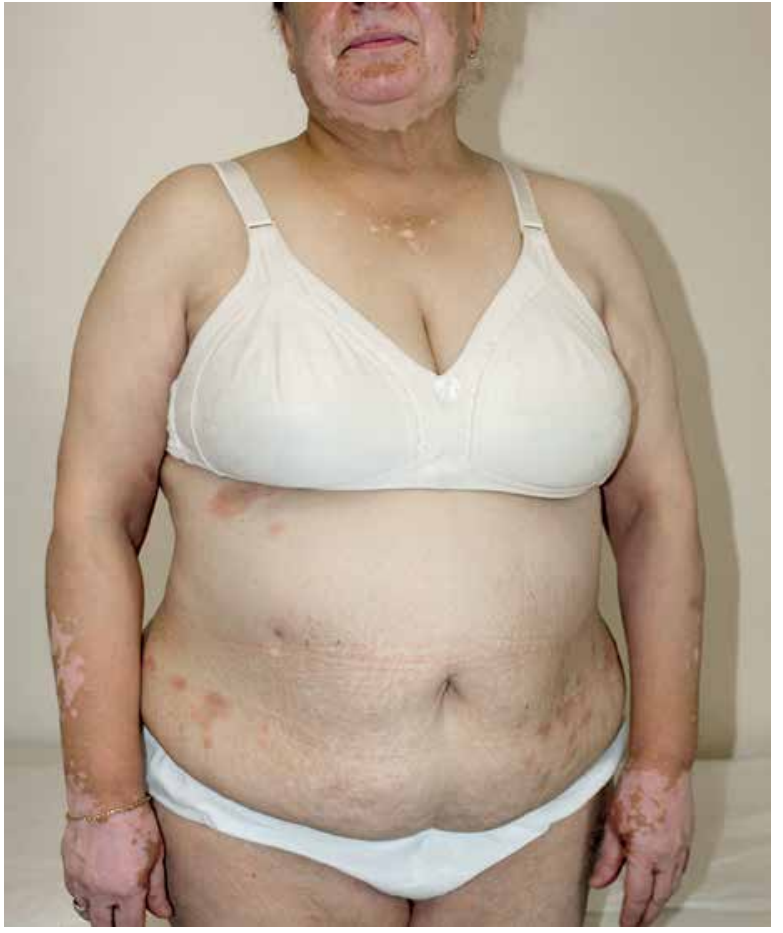

Figure 1. Depigmented patches and indurated dusky violaceous patches

has been proposed, which includes circumscribed, linear, generalized, pansclerotic and mixed types owing to the fact that the former one does not encompass the cases present with more than one clinical variant of morphea and also covers many diagnoses that would be out of the morphea spectrum. Generalized morphea is an uncommon type of morphea, in which more than four lesions greater than $3 \mathrm{~cm}$ in diameter arise in two or more body sites $[1,2,4,5]$.

In the literature there are several case reports implicating autoimmunity as the underlying cause in the development of morphea [6-11]. These reports clearly indicate the association of morphea with various autoimmune diseases, including primary biliary cirrhosis [6], myasthenia gravis [11], Hashimoto's thyroiditis [9] and multiple autoimmune syndrome (MAS) [10]. Moreover, it has been shown that cytokines and soluble receptors including interleukin-2 (IL-2), IL-4, IL-6, soluble interleukin-2 (sIL-2) receptor and SIL-6 receptor levels increase in patients with morphea. In addition, autoantibodies such as ANA, anti-single-stranded DNA (ssDNA) and anti-histone antibody have been found at higher frequencies in these patients. While these findings strongly highlight morphea as an organ-specific autoimmune disorder, generalized morphea is the most implicated one with the autoimmune background [5].

Vitiligo is a common acquired pigmentary disorder characterized by well-demarcated depigmented patches. The relation of vitiligo with autoimmune diseases, and

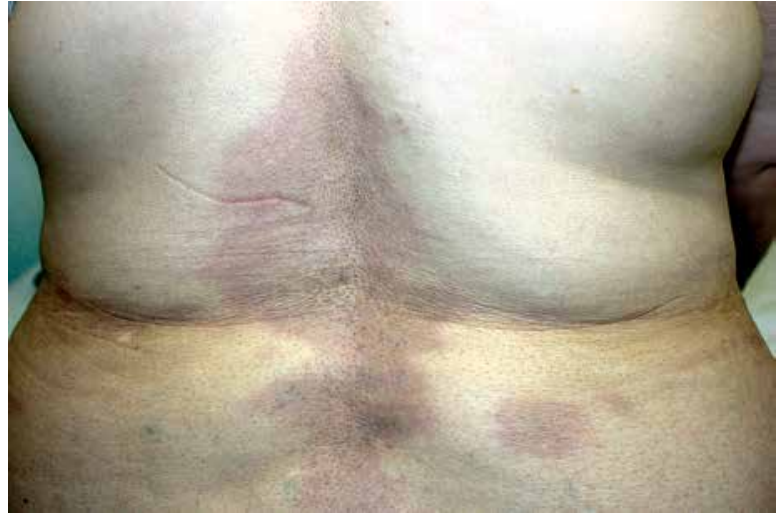

Figure 2. Mauve-coloured indurated plaques on the back

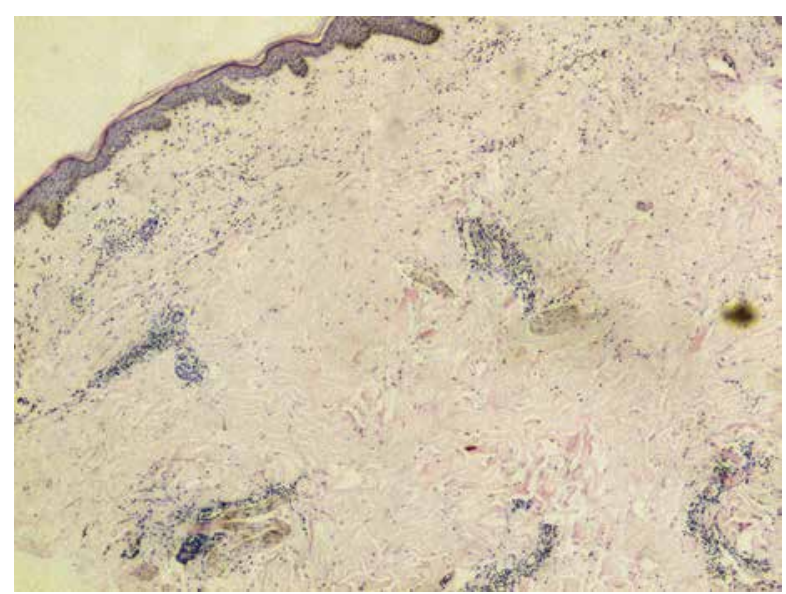

Figure 3. Superficial and deep dermal perivascular lymphocytic infiltrate and focally increased dermal collagen $(H+E, 4 x)$

detection of organ-specific autoantibodies in vitiligo patients, also the association of particular alleles of the multilocus major histocompatibility complex (MHC) with susceptibility to vitiligo hypothetically suggest that vitiligo is an autoimmune disorder [3, 12,13]. Although both morphea and vitiligo are autoimmune diseases, concurrent appearance of these entities have rarely been reported [7, 9, 10, 14, 15]. Few reports in the literature have presented cases with MAS [10], cirrhosis [7] and Hashimoto's thyroiditis [9] in patients with both vitiligo and morphea. Here, we report a concurrent appearance of vitiligo and morphea, which are two skin diseases of autoimmune origin in a patient with Hashimoto's thyroiditis. To the best of our knowledge, our case is the second report in the literature describing morphea, vitiligo and Hashimoto's thyroiditis association. This unique presentation clearly suggests an autoimmune phenomenon in the pathogenesis of these entities. We think that case reports like ours would provide better understanding of underlying mechanisms of these diseases and the existing knowledge dealing with the relationship between 
autoimmunity and its implication in the pathogenesis of morphea and vitiligo should be further revised and assessed.

\section{Conflict of interest}

The authors declare no conflict of interest.

\section{References}

1. Fett N, Werth VP. Update on morphea: part I. Epidemiology, clinical presentation, and pathogenesis. J Am Acad Dermatol 2011; 64: 217-28.

2. Goodfield MJD, Jones SK, Veale DJ. The connective tissue diseases. In: Rook's Textbook of Dermatology. 8th ed. Burns T, Breathnach S, Cox N, Grittiths C (eds.). Wiley-Blackwell, Oxford 2010; 51.64-80.

3. Bowcock AM, Fernandez-Vina M. Targeting skin: vitiligo and autoimmunity. J Invest Dermatol 2012; 132: 13-5.

4. Fett N. Scleroderma: nomenclature, etiology, pathogenesis, prognosis, and treatments: facts and controversies. Clin Dermatol 2013; 31: 432-7.

5. Takehara K, Sato S. Localized scleroderma is an autoimmune disorder. Rheumatology (Oxford) 2005; 44: 274-9.

6. González-López MA, Drake M, González-Vela MC, et al. Generalized morphea and primary biliary cirrhosis coexisting in a male patient. J Dermatol 2006; 33: 709-13.

7. Soylu S, Gül U, Gönül M, et al. An uncommon presentation of the co-existence of morphea and vitiligo in a patient with chronic hepatitis $B$ virus infection: is there a possible as sociation with autoimmunity? Am J Clin Dermatol 2009; 10: 336-8.

8. Khalifa M, Ben Jazia E, Hachfi W, et al. Autoimmune hepatitis and morphea: a rare association. Gastroenterol Clin Biol 2006; 30: 917-8.

9. Dervis E, Acbay O, Barut G, et al. Association of vitiligo, morphea, and Hashimoto's thyroiditis. Int I Dermatol 2004; 43: 236-7.

10. Bonilla-Abadía F, Muńoz-Buitrón E, Ochoa CD, et al. A rare association of localized scleroderma type morphea, vitiligo, autoimmune hypothyroidism, pneumonitis, autoimmune thrombocytopenic purpura and central nervous system vasculiti. Case report. BMC Res Notes 2012; 5: 689.

11. Parra V, Driban N, Bassotti A. Localized morphea and myasthenia gravis. J Am Acad Dermatol 2008; 49: 1.

12. Ongenae K, Van Geel N, Naeyaert JM. Evidence for an autoimmune pathogenesis of vitiligo. Pigment Cell Res 2003; 16: $90-100$

13. Czajkowski R, Męcińska-Jundziłł K. Current aspects of vitiligo genetics. Postep Derm Alergol 2014; 31: 247-55.

14. Yadav P, Garg T, Chander R, Nangia A. Segmental vitiligo with segmental morphea: an autoimmune link? Indian Dermatol Online I 2014; 5: S23-5.

15. Bonifati C, Impara G, Morrone A, et al. Simultaneous occurrence of linear scleroderma and homolateral segmental vitiligo. J Eur Acad Dermatol Venereol 2006; 20: 63-5. 